This item was submitted to Loughborough's Research Repository by the author.

Items in Figshare are protected by copyright, with all rights reserved, unless otherwise indicated.

\title{
Generation of solitary waves by transcritical flow over a step
}

PLEASE CITE THE PUBLISHED VERSION

LICENCE

CC BY-NC-ND 4.0

\section{REPOSITORY RECORD}

Grimshaw, Roger H.J., D.H. Zhang, and K.W. Chow. 2019. "Generation of Solitary Waves by Transcritical Flow over a Step". figshare. https://hdl.handle.net/2134/2612. 


\title{
Generation of solitary waves by transcritical flow over a step
}

\author{
By R.H.J. G R IMSH A W', D. - H. Z Z A N G ${ }^{2}$ \\ AND K.W. CHO W ${ }^{2}$ \\ ${ }^{1}$ Department of Mathematical Sciences, Loughborough University, Loughborough, LE11 3TU, \\ UK \\ ${ }^{2}$ Department of Mechanical Engineering, University of Hong Kong, Pokfulam Road , Hong \\ Kong
}

(Received ?? and in revised form ??)

It is well-known that transcritical flow over a localised obstacle generates upstream and downstream nonlinear wavetrains. The flow has been successfully modeled in the framework of the forced Korteweg-de Vries equation, where numerical and asymptotic analytical solutions have shown that the upstream and downstream nonlinear wavetrains have the structure of unsteady undular bores, connected by a locally steady solution over the obstacle, which is elevated on the upstream side and depressed on the downstream side. In this paper we consider the analogous transcritical flow over a step, primarily in the context of water waves. We use numerical and asymptotic analytical solutions of the forced Korteweg-de Vries equation, together with numerical solutions of the full Euler equations, to demonstrate that a positive step generates only an upstream-propagating undular bore, and a negative step generates only a downstream-propagating undular bore.

\section{Introduction}

The flow of a fluid over an obstacle is a classical and fundamental problem in fluid mechanics. Our concern here is with the upstream and downstream waves that may be generated. The most well-known scenario is for free-surface flow, when the allowed waves are water waves, but the same essential features arise in many other physical systems, such as the flow over a density-stratified fluid over topography when the relevant waves are internal waves. When the flow is not critical, that is, the imposed flow speed does not close to any linear long wave speed, linear theory may be used to describe the wave field, and typically the full solution can be obtained using Fourier transforms, followed by classical phase and group velocity arguments (see, for instance, Lighthill (1978) and Whitham (1974)). For instance, in the case of water waves, typically stationary lee waves are found downstream in subcritical flow (that is, the flow speed $U<c$, the linear long wave speed), together with transients propagating both upstream and downstream, while only downstream-propagating transients are found in supercritical flow $(U>c)$. However, these linear solutions fail near criticality $(U=c)$, as then the wave energy cannot propagate away from the obstacle. In this case it is necessary to invoke weak nonlinearity to obtain a suitable theory, and it is now well established that the forced Korteweg-de Vries (fKdV) equation is an appropriate model.

For water waves on an undisturbed depth $h$, the fKdV equation is

$$
-A_{t}-\Delta A_{x}+\mu A A_{x}+\lambda A_{x x x}+\frac{c}{2} F_{x}=0,
$$




$$
\text { where } \mu=\frac{3 c}{2 h}, \lambda=\frac{c h^{2}}{6}, c=(g h)^{1 / 2} \text {. }
$$

Here $A(x, t)$ is the wave amplitude, here the surface elevation above the undisturbed depth $h$, while $F(x)$ is the obstacle profile and $\Delta=U-c$ is the criticality parameter $(\Delta<$ $0(>0)$ is the subcritical (supercritical) regime). The fKdV equation has been derived for water waves by Akylas (1984), Cole (1985), Mei (1986), Wu (1987), Lee et al (1989), and for internal waves by Grimshaw \& Smyth (1986) and Melville \& Helfrich (1987) where the coefficients $\mu, \lambda$ and the speed $c$ are then given by expressions involving the modal function for the relevant internal wave mode. Note that for water waves, the forcing provided by a bottom obstacle, or by an applied surface pressure field, or by a slender ship, are equivalent in the weakly nonlinear approximation. Numerical and aysmptotic analytical solutions of the fKdV equation by these and other authors have demonstrated that for flow over a localized obstacle, in the transcritical regime the solution typically consists of an upstream and downstream nonlinear wavetrains, connected by a locally steady solution over the obstacle which is elevated on the upstream side and depressed on the downstream side. These nonlinear wavetrains have the structure of unsteady undular bores. Near exact criticality, the upstream wavetrain is attached to the obstacle, and to a high degree of approximation, consists of upstream propagating solitary waves.

These theoretical predictions were anticipated in several laboratory experiments. For water waves the first reported observations of the upstream waves generated by a steadily moving ship were apparently made by Thews \& Landweber $(1934,1935)$. Systematic experiments reported by Huang et al (1982), Ertekin et al (1984) and Lee et al (1989) established the presence of upstream propagating solitary waves. As well as the numerical simulations of the fKdV equation, simulations of a generalized Boussinesq equations by $\mathrm{Wu} \& \mathrm{Wu}(1982)$ and Lee et al (1989), and of a Green-Naghdi model by Ertekin et al (1986) also confirmed the generation of upstream propagating solitary waves by transcritical flow over an obstacle.

Recently Zhang \& Chwang (2001) simulated the full Euler equations for transcritical flow over an obstacle, and as well as confirming the basic scenario of the generation of upstream and downstream undular bores, found good agreement with the theory of Grimshaw \& Smyth (1986) based on the fKdV equation. A particular feature of their numerical simulations was the exploration of the effect of the width of the obstacle, and in a limiting configuration they simulated transcritical flow over either a positive (forward-facing) step, or over a negative (backward-facing )step. Their results showed that a positive step generates an upstream-propagating undular bore, and a negative step generates a downstream-propagating undular bore, thus suggesting that the upstream and downstream wavetrains generated by transcritical flow over a localized obstacle may be generated by separate proceses. It is these simulations which have lead to the present investigation, where we seek an explanation for this behaviour in the framework of the fKdV equation (1.1), using both theoretical asymptotic solutions constructed using the techniques of Grimshaw \& Smyth (1986) and numerical simulations.

In nondimensional form, based on the velocity and length scales $c, h$ equation (1.1) becomes

$$
-A_{t}-\Delta A_{x}+\frac{3}{2} A A_{x}+\frac{1}{6} A_{x x x}+\frac{1}{2} F_{x}=0 .
$$

Here the nondimensional $\Delta=F r-1$ where $F r=U / c$ is the Froude number. The fKdV equation in canonical form is obtained by putting

$$
t^{*}=\frac{1}{6} t, A^{*}=\frac{3}{2} A, F^{*}=\frac{9}{2} F, \Delta^{*}=6 \Delta .
$$


Omitting the superscripts we get

$$
-A_{t}-\Delta A_{x}+6 A A_{x}+A_{x x x}+F_{x}(x)=0 .
$$

This is to be solved with the initial condition that $A(x, 0)=0$, which corresponds to the introduction of the topographic obstacle for $t \geqslant 0$. In a laboratory reference frame, this is equivalent to the situation in which the obstacle is at rest for $t<0$ and is then moved at speed $U$ to the left for $t>0$. Our interest is the case when the forcing term represents a step, that is $F(x)=0$ for $x<0$ and then varies monotonically for $0<x<W$ to a value $F_{M}>0(<0)$ for $x>W$, corresponding to a positive (negative) step. In section 2 we construct asymptotic solutions in the spirit of Grimshaw \& Smyth (1986)), and in section 3 we describe some numerical simulations of the fKdV equation, and also of the full Euler equations for comparison.

\section{Asymptotic solutions of the forced Korteweg-de Vries equation}

\subsection{Critical flow over a localized obstacle}

Before considering the main case of interest, that is flow over a step, it is useful to present a summary of the theory for flow over a localized obstacle, based on Grimshaw \& Smyth (1986) and Smyth (1987). First we recall the typical solution of (1.5) when the forcing $F(x)$ is positive and localized. That is, $F(x)$ is positive, and non-zero only in a vicinity of $x=0$, with a maximum value of $F_{M}>0$. The solution is characterised by upstream and downstream wavetrains connected by a locally steady solution over the obstacle. For subcritical flow $(\Delta<0)$ the upstream wavetrain weakens, and for sufficiently large $|\Delta|$ detaches from the obstacle, while the downstream wavetrain intensifies and for sufficiently large $|\Delta|$ forms a stationary lee wave field. On the other hand, for supercritical flow $(\Delta>0)$ the upstream wavetrain develops into well-separated solitary waves while the downstream wavetrain weakens and moves further downstream (for more details see Grimshaw and Smyth 1986 and Smyth 1987). The origin of the upstream and downstream wavetrains can be found in the structure of the locally steady solution over the obstacle. In the transcritical regime this is characterised by a transition from a constant state $A_{-}$upstream $(x<0)$ of the obstacle to a constant state $A_{+}$downstream $(x>0)$ of the obstacle, where $A_{-}<0$ and $A_{+}>0$. It is readily shown that $\Delta=3\left(A_{+}+A_{-}\right)$ independently of the details of the forcing term $F(x)$. Explicit determination of $A_{+}$and $A_{-}$requires some knowledge of the forcing term $F(x)$. However, in the "hydraulic" limit when the linear dispersive term in (1.5) can be neglected, it is readily shown that, for all localised $F(x)$,

$$
6 A_{ \pm}=\Delta \mp\left(12 F_{M}\right)^{1 / 2} .
$$

This expression also serves to define the transcritical regime, which is

$$
|\Delta|<\left(12 F_{M}\right)^{1 / 2} .
$$

Thus upstream of the obstacle there is a transition from the zero state to $A_{-}$, while downstream the transition is from $A_{+}$to 0 ; each transition is effectively generated at $X=0$. Note that in the unscaled form (1.3) the regime (2.2) becomes $|\Delta|<\left(3 F_{M} / 2\right)^{1 / 2}$.

Both transitions are resolved by "undular bore" solutions as described in the Appendix. That in $x<0$ is exactly described by (A 3) to (A 6) with $x$ replaced by $\Delta t-x$, and $A_{0}$ by $A_{-}$. It occupies the zone

$$
\Delta-4 A_{-}<\frac{x}{t}<\max \left\{0, \Delta+6 A_{-}\right\} .
$$

Note that this upstream wavetrain is constrained to lie in $x<0$, and hence is only fully 
realised if $\Delta<-6 A_{-}$. Combining this criterion with (2.1) and (2.2) defines the regime

$$
-\left(12 F_{M}\right)^{1 / 2}<\Delta<-\frac{1}{2}\left(12 F_{M}\right)^{1 / 2},
$$

where a fully developed undular bore solution can develop upstream. On the other hand, the regime $\Delta>-6 A_{-}$or

$$
-\frac{1}{2}\left(12 F_{M}\right)^{1 / 2}<\Delta<\left(12 F_{M}\right)^{1 / 2},
$$

is where the upstream undular bore is only partially formed, and is attached to the obstacle. In this case the modulus $m$ of the Jacobian elliptic function varies from 1 at the leading edge (thus describing solitary waves) to a value $m_{-}(<1)$ at the obstacle, where $m_{-}$can be found from (A 5) by replacing $x$ with $\Delta t$ and $A_{0}$ with $A_{-}$.

The transition in $x>0$ can also be described by (A 3) to (A 6) where we now replace $x$ with $\left(\Delta+6 A_{+}\right) t-x, A_{0}$ with $-A_{+}$, and $d$ with $d-A_{+}$. This "undular bore" solution occupies the zone

$$
\max \left\{0, \Delta-2 A_{+}\right\}<\frac{x}{t}<\Delta-12 A_{+} .
$$

Here, this downstream wavetrain is constrained to lie in $x>0$, and hence is only fully realised if $\Delta>2 A_{+}$. Combining this criterion with (2.1) and (2.2) defines the regime (2.5), and so a fully detached downstream undular bore coincides with the case when the upstream undular bore is attached to the obstacle. On the other hand, in the regime (2.4), when the upstream undular bore is detached from the obstacle, the downstream undular bore is attached to the obstacle, with a modulus $m_{+}(<1)$ at the obstacle, where $m_{+}$can be founding from (A 5) by replacing $x$ with $\Delta-6 A_{+}$and $A_{0}$ with $A_{+}$. Indeed now a stationary lee wavetrain develops just behind the obstacle (for further details, see Smyth, 1987).

For the case when the obstacle has negative polarity (that is $F(x)$ is negative, and non-zero only in the vicinity of $x=0$ ), the upstream and downstream solutions are qualitatively similar. However, the solution in the vicinity of the obstacle remains transient, and this causes a modulation of the "undular bore" solutions.

\subsection{Critical flow over a step}

Here we consider positive and negative steps, where

$$
\begin{aligned}
& F(x)=0, \quad \text { for } \quad 0<x<W, \\
& F(x)=F_{M}, \quad \text { for } \quad x>W,
\end{aligned}
$$

and $F(x)$ varies monotonically in $0<x<W$. A positive (negative) step has $F_{M}>0(<$ 0 ). Strictly $F(x)$ should return to zero for some $L>>W$. Here we ignore this, and in effect assume that $L \rightarrow \infty$. In practice it means that the solutions constructed below are only valid for some limited time, determined by how long it takes for a disturbance to travel the distance $L$.

We shall sketch how the solution for the localized forcing described above becomes modified for a step. Adapting the approach used by Grimshaw and Smyth (1986) the first step is to construct the local steady-state solution in the forcing region, $0<x<L$, that is $A=A(x), 0<x<L$ and

$$
\begin{aligned}
& A=A_{-} \quad \text { for } \quad x<0, \\
& A=A_{+} \quad \text { for } \quad x>W .
\end{aligned}
$$

In the the hydraulic limit when the dispersive term in (1.5) is omitted, it is readily found 
that

$$
-\Delta A+3 A^{2}+F=C .
$$

Here the constant $C$ is determined by considering the long-time limit of the unsteady hydraulic solution, as in Grimshaw and Smyth (1986). But note that

$$
C=-\Delta A_{-}+3 A_{-}^{2}=-\Delta A_{+}+3 A_{+}^{2}+F_{M},
$$

giving a connection between $A_{-}$and $A_{+}$.

Suppose first that the step is positive, $F_{M}>0$. Then, as in Grimshaw \& Smyth (1986), the local hydraulic solution is found by omitting the linear dispersive term in (A 1) and then solving the initial value problem for the resulting first-order hyperbolic equation by using characteristics and so determining the long-time solution. Omitting details, we find that the local hydraulic solution is:

$$
\begin{aligned}
\Delta \leqslant 0: & 6 A_{-}=\Delta+\left(\Delta^{2}+12 F_{M}\right)^{1 / 2}, \quad 6 A_{+}=0, \\
0<\Delta<\left(12 F_{M}\right)^{1 / 2}: & 6 A_{-}=\Delta+\left(12 F_{M}\right)^{1 / 2}, \quad 6 A_{+}=\Delta, \\
\Delta>\left(12 F_{M}\right)^{1 / 2}: & 6 A_{-}=0 \quad 6 A_{+}=\Delta-\left(\Delta^{2}-12 F_{M}\right)^{1 / 2}
\end{aligned}
$$

Here the constant in (2.10) is $C=F_{M}, F_{M}-\Delta^{2} / 12,0$ resepctively.

In all cases, the upstream solution $A_{-}>0$ is a "shock" in the hydraulic limit (although in (2.13) the shock has zero strength and so can be ignored), which needs to be replaced with an "undular bore" as in section 2 . That is, the undular bore is again given by (A 3) to (A 6) with $x$ replaced by $\Delta t-x$ and $A_{0}$ by $A_{-}$and occupies the zone (compare (2.3))

$$
\Delta-4 A_{-}<\frac{x}{t}<\max \left\{0, \Delta+6 A_{-}\right\} .
$$

But now $A_{-}$is given by $(2.11,2.12)$ in place of $(2.1)$. For a fully detached undular bore, $\Delta+6 A_{-}<0$, and combining this criterion with $(2.11,2.12,2.13)$, we get the regime

$$
\Delta<-2\left(F_{M}\right)^{1 / 2}<0 .
$$

On the other hand the regime where $\Delta+6 A_{-}>0$ but $\Delta-4 A_{-}<0$, or

$$
-2\left(F_{M}\right)^{1 / 2}<\Delta<\left(12 F_{M}\right)^{1 / 2},
$$

is where the upstream undular bore is only partially formed and is attached to the obstacle. Note that the regimes $(2.15,2.16)$ for the unscaled equation $(1.3)$ are $\Delta<$ $-\left(F_{M} / 2\right)^{1 / 2}<0$ and $-\left(F_{M} / 2\right)^{1 / 2}<\Delta<\left(3 F_{M} / 2\right)^{1 / 2}$ respectively.

Downstream, for $0<\Delta<\left(12 F_{M}\right)^{1 / 2}$ the hydraulic solution with $A_{+}=\Delta>0$ is terminated by a rarefraction wave, and so no undular bore solution is needed. Instead a weak oscillatory wave train is needed to smooth the corners. For $\Delta>\left(12 F_{M}\right)^{1 / 2}$ (or $\Delta>\left(3 F_{M} / 2\right)^{1 / 2}$ in unscaled variables) there is no upstream disturbance, and again $A_{+}>0$ so that a rarefraction wave is needed. In the present weakly nonlinear limit, this agrees with the numerical results of King \& Bloor (1987) who computed steadystate flows over a step. In this regime, they found a class of supercritical steady flows for which, in our notation, $A_{-}=0, A_{+}>0$, in agreement with (2.13). For $\Delta<0$ the hydraulic limit (2.13) predicts a zero disturbance downstream. However, in this regime, there is an expectation that stationary lee waves may form, but this of course cannot be predicted in the hydraulic limit. In this context we note that for subcritical flows with $\Delta<-\left(3 F_{M} / 2\right)^{1 / 2}$ (in unscaled variables) King \& Bloor (1987) found a class of flows with $A_{-}=0$ upstream, but with steady lee waves downstream, riding on a depressed level. In our notation, there is indeed a corresponding steady hydraulic solution with $A_{-}=0,6 A_{+}=\Delta+\left(\Delta^{2}-12 F_{M}\right)^{1 / 2}<0$, and we might expect that stationary lee 
waves could form on this depressed level. However, the present initial-value problem for the hydraulic solution shows that this state cannot be reached, and instead the outcome is (2.13). Further, our numerical solutions of the fKdV equation, and of the full Euler equations, discussed in the next section, do not find any evidence for the formation of steady lee waves downstream of the step.

Next consider the negative step, $F_{M}<0$, for which the local hydraulic solution is:

$$
\begin{aligned}
\Delta \geqslant 0: & 6 A_{+}=\Delta-\left(\Delta^{2}-12 F_{M}\right)^{1 / 2}, 6 A_{-}=0, \\
-\left(-12 F_{M}\right)^{1 / 2}<\Delta<0: & 6 A_{+}=\Delta-\left(-12 F_{M}\right)^{1 / 2}, 6 A_{-}=\Delta, \\
\Delta<-\left(-12 F_{M}\right)^{1 / 2}: & 6 A_{+}=0,6 A_{-}=\Delta-\left(\Delta^{2}+12 F_{M}\right)^{1 / 2} .
\end{aligned}
$$

Here the constant in (2.10) is $C=0,-\Delta^{2} / 12, F_{M}$ resepctively. In all cases the downstream solution $A_{+}<0$ is a shock (in (2.19) the shock has zero strength), and needs to be replaced by an undular bore solution. Now the undular bore is given by (A 3) to (A 6) with $x$ replaced by $\left(\Delta+6 A_{+}\right) t-(x-W), A_{0}$ by $-A_{+}$and $d$ with $d-A_{+}$. It occupies the zone (compare (2.6))

$$
\max \left\{0, \Delta-2 A_{+}\right\}<\frac{x-W}{t}<\Delta-12 A_{+} .
$$

where $A_{+}$is given by $(2.17,2.18)$. For a fully detached undular bore, $\Delta-2 A_{+}>0$, and combining with the criteria $(2.18,2.19)$ we get the regime

$$
\Delta>-\left(-3 F_{M}\right)^{1 / 2} .
$$

On the other hand, the regime where $\Delta-2 A_{+}<0$ but $\Delta-12 A_{+}>0$, or

$$
-\left(-12 F_{M}\right)^{1 / 2}<\Delta<-\left(-3 F_{M}\right)^{1 / 2}<0 .
$$

is where the undular bore is only partially formed. For $\Delta<-\left(-12 F_{M}\right)^{1 / 2}$ we expect a stationary lee-wave train to form downstream. For the original unscaled equation (1.3) the regime $(2.21,2.22)$ becomes $\Delta>-\left(-3 F_{M} / 8\right)^{1 / 2}$ and $-\left(-3 F_{M} / 2\right)^{1 / 2}<\Delta<$ $-\left(-3 F_{M} / 8\right)^{1 / 2}$ respectively.

For $\Delta<0$ the upstream solution, $A_{-}<0$, is terminated by a rarefraction wave and no shock is needed, but an oscillatory wave train is needed to smooth out the corners. For $\Delta>0$ the upstream solution is zero.

\section{Numerical results}

3.1. Numerical simulation of the forced Korteweg-de Vries equation

The fKdV equation (1.3) is solved numerically by a finite difference scheme. We use a leapfrog scheme in time and central differencing in space,

$$
\begin{aligned}
\frac{\partial A}{\partial t} & =\frac{A_{i}^{n+1}-A_{i}^{n-1}}{2 \delta t}+O\left((\delta t)^{2}\right), \\
A \frac{\partial A}{\partial x} & =\frac{1}{3}\left(A_{i+1}^{n}+A_{i}^{n}+A_{i-1}^{n}\right) \frac{A_{i+1}^{n}-A_{i-1}^{n}}{2 \delta x}+O\left((\delta x)^{2}\right), \\
\frac{\partial^{3} A}{\partial x^{3}} & =\frac{A_{i+2}^{n}-2 A_{i+1}^{n}+2 A_{i-1}^{n}-A_{i-2}^{n}}{2(\delta x)^{3}}+O\left((\delta x)^{2}\right),
\end{aligned}
$$

where we use a subscript to denote the spatial location and a superscript to denote the time level. Substituting these finite difference approximations back into the fKdV 
equation (1.3), we obtain an explicit scheme as follows,

$$
\begin{aligned}
A_{i}^{n+1}= & A_{i}^{n-1}-\frac{\delta t}{\delta x}\left(\Delta-\frac{A_{i+1}^{n}+A_{i}^{n}+A_{i-1}^{n}}{2}\right)\left(A_{i+1}^{n}-A_{i-1}^{n}\right) \\
& +\frac{\delta t}{6(\delta x)^{3}}\left(A_{i+2}^{n}-2 A_{i+1}^{n}+2 A_{i-1}^{n}-A_{i-2}^{n}\right)+\delta t\left(F_{x}\right)_{i}^{n} .
\end{aligned}
$$

The scheme (3.4) is second order accurate in time and space, and is conditionally stable. Typical $\delta t$ and $\delta x$ used in our computations are $\delta t=0.001$ and $\delta x=0.1$.

The forcing takes the form

$$
F(x)=\frac{F_{M}}{2}(\tanh \gamma x-\tanh \gamma(x-L)),
$$

where $F_{M}>0$ is the height of the step (see (2.7)), $L$ is the separation between the front and the rear steps, and $1 / \gamma$ measures the width of the steps (i.e. a measure of $W$ in (2.7)). Note that this form of forcing enables us to examine both a positive step at $x=0$ and a negative step at $x=L$ in the same simulation, at least until the time it takes for a disturbance to travel a distance $L$ across the step.

\subsection{Numerical simulation of the Euler equations}

The following summary is based on Zhang and Chwang (1999), reduced to the case of an of an inviscid fluid. The motion of an incompressible inviscid fluid under the influence of gravity is governed by the Euler equations and the equation of continuity, which in a body- and free-surface-fitted curvilinear coordinate system $\left(\xi^{i}\right)$ have the form

$$
\begin{gathered}
\frac{\partial}{\partial t}\left(\frac{u_{i}}{J}\right)+\frac{\partial}{\partial \xi^{j}}\left(\frac{u_{i}}{J} \frac{\partial \xi^{j}}{\partial t}+\frac{u_{i} V^{j}}{J}\right)=-\frac{\partial}{\partial \xi^{j}}\left(\frac{\phi}{J} \frac{\partial \xi^{j}}{\partial x_{i}}\right), \\
\frac{\partial}{\partial \xi^{j}}\left(\frac{V^{j}}{J}\right)=0 \\
\text { where } J=\frac{\partial\left(\xi^{1}, \xi 2\right)}{\partial\left(x_{1}, x_{2}\right)}, \quad V^{j}=\frac{\partial \xi^{j}}{\partial x_{k}} u_{k}, \quad \phi=p+\frac{x_{2}}{F r^{2}} .
\end{gathered}
$$

Here $x_{i}$ is a reference Cartisian coordinate system, $u_{i}$ is the velocity component in the $i$-direction, $p$ is the pressure, $t$ is the time, $J$ is the Jacobian of the transformation and $V^{j}$ is the contravariant velocity component. The Euler equations are normalized by the undisturbed water depth $h$ and the upstream velocity $U$. The pressure $p$ is nondimensionalized by $\rho U^{2}$, time by $h / U$, the Froude number is defined here by $F r=U / \sqrt{g h}$, and $\rho$ is the constant density. Note that this nondimensionalization differs from that used for the fKdV equation (1.5) where velocities were scaled by $c$, but the difference is small for transcritical flow. If $\eta\left(x_{1}, t\right)$ is the free-surface elevation, the kinematic condition is

$$
\frac{\partial \eta}{\partial t}+u_{1} \frac{\partial \eta}{\partial x_{1}}=u_{2}, \quad \text { at } \quad x_{2}=\eta
$$

In the absence of surface tension, the dynamic condition on the free surface is

$$
p=0 \quad \text { at } \quad x_{2}=\eta \text {. }
$$

A slip boundary condition is imposed on the bottom, and a Neumann-type boundary condition is imposed at the downstream boundary.

These governing equations are discretized on a regular grid by a finite difference method. The velocity and pressure are evaluated at the computational cell centres. Spatial derivative are discretized using a second-order central difference, and the QUICK 


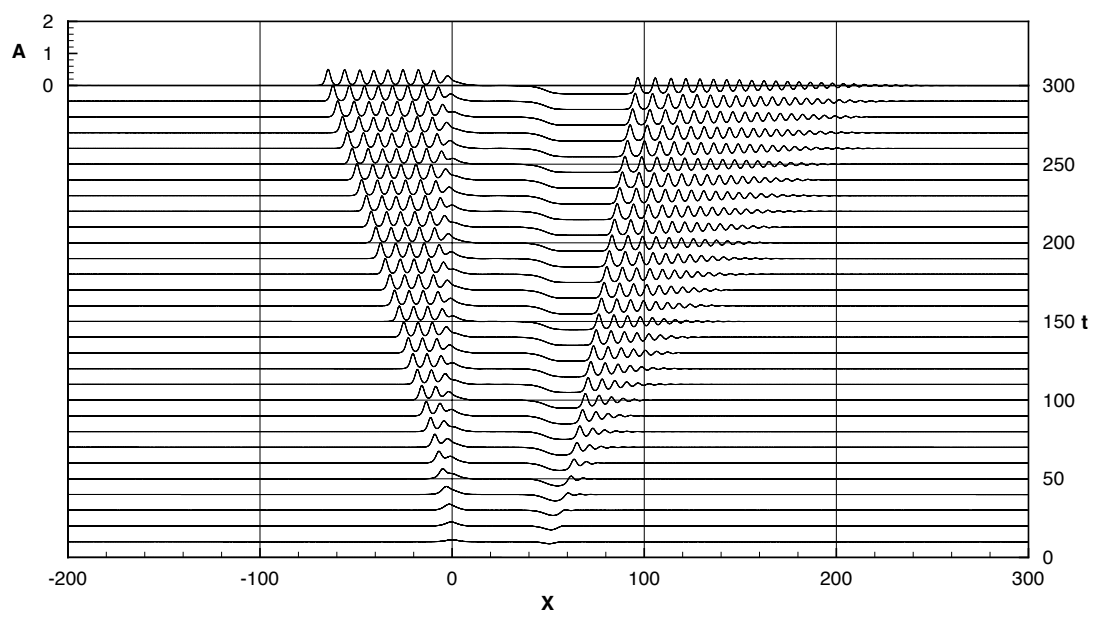

Figure 1. Numerical simulation of the fKdV equation (1.3) for $F_{M}=0.1, \gamma=0.25, L=50, \Delta=0.0$.

scheme is used for discretization of the convection terms. Time marching is carried out using a time-splitting fractional step. It is a two-step predictor-corrector scheme. In the predictor step, an intermediate velocity field $\tilde{u}_{i}$ is computed explicitly by integrating equation (3.6) in time using the velocity and pressure from the previous time level $n$. Then the location of the free surface at the time level $n+1$ is evaluated by integrating equation (3.9) explicitly, and the grid of the flow domain is re-generated to fit the newly determined free surface. Requiring the velocity field $u_{i}^{n+1}$ to satisfy the continuity equation, a Poisson equation is obtained for solving the pressure increment $\delta \phi=\phi^{n+1}-\phi^{n}$, with the intermediate velocity field $\tilde{u}_{i}$ as the source term. The pressure field at the time level $n+1$ is then updated, and the velocity field $u_{i}^{n+1}$ is obtained by modifying the intermediate velocity field $\tilde{u}_{i}$ using the pressure increment.

The solution domain in the vertical direction is of one unit length and 20 grid points are evenly distributed. In the horizontal direction, the solution domain is made sufficiently large so that the upstream wavetrain does not reach the upstream boundary at the final time of the computations. Thus, far upstream the flow is uniform and the free surface is undisturbed. Typically, 400 unit lengths are used and 600 grid points are distributed in the horizontal direction. The forcing is located in the middle of the solution domain $\left(x_{1}=0\right)$. The grid is refined in the forcing region $\left(\delta x_{1} \approx 0.5\right)$ and gradually coarsened towards the two ends of the solution domain. The coarse grid in the region near the downstream boundary acts as a dissipation zone to prevent the reflection of waves into the solution domain. The time step is set to be $\delta t=0.01$. The computation starts at the initial condition of zero velocity and a flat free surface.

\subsection{Description of results}

In Figures 1 to 3 we show the simulations of the fKdV equation (1.3) for $F_{M}=0.1, \gamma=$ $0.25, L=50$ and $\Delta=0.0,0.2,-0.2$ respectively. The corresponding simulations for the 


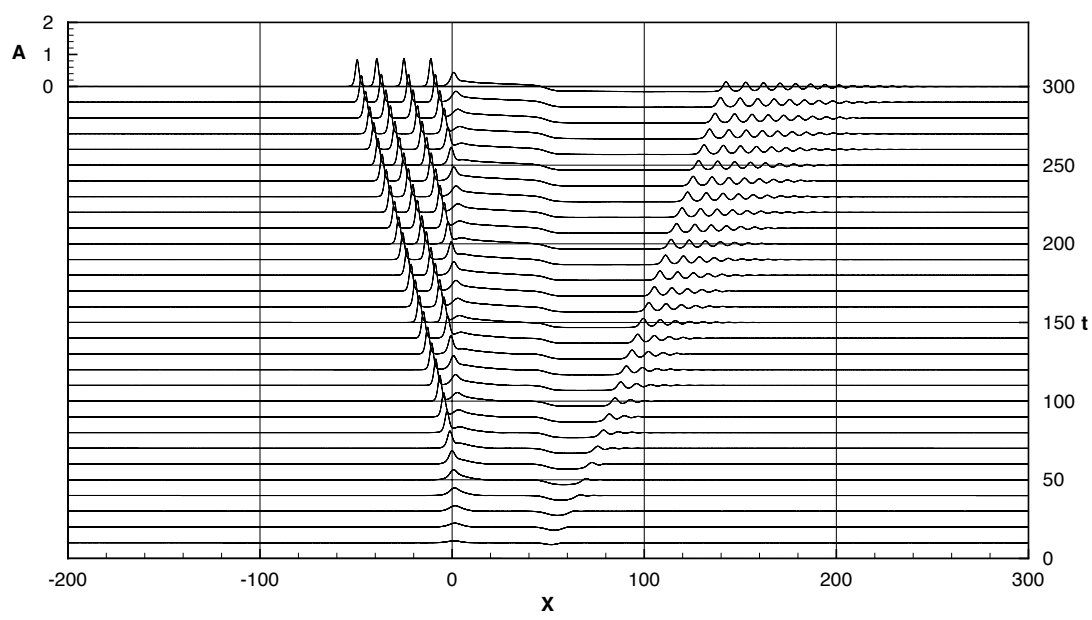

Figure 2. As for Figure 1, but with $\Delta=0.2$

Euler equations are shown in Figures 4 to 6, and a quantitative comparison between our two sets of simulations and the theory described in section 2 is shown in Table 1 . In this table we compare the key features $A_{-}$(the upstream elevation at the step at $\left.x=0\right), A_{+}$ (the downstream depression at the step at $x=50$ ), $A_{w-}$ (the amplitude of the leading wave upstream) and $A_{w-}$ (the amplitude of the leading wave downstream). Note that in the present theory $A_{w-}=2 A_{-}$and $A_{w+}=-2 A_{+}$(see the Appendix).

First we consider the results for the fKdV equation. When $\Delta=0$ (Figure 1) we see that an upstream undular bore is attached to the positive step at $x=0$, and a downstream undular bore propagates away from the negative step at $x=50$, leaving a depression zone behind. Further there is no evidence of a disturbance generated over the step. This scenario is in complete accord with our theoretical predictions. A quantitative comparison is displayed in Table 1 where we see that there is excellent agreement. The largest discrepancy is for $A_{-}$upstream, which is the hardest quantity to estimate from the numerical solution as the upstream undular bore is attached to the step at $x=0$. In this case of exact resonance the hydraulic limit predicts that the entire solution outside the step is exactly the same as that for flow over a localized positive forcing; indeed we see that $A_{-}=\left(12 F_{M}\right)^{1 / 2}$ (see $(2.11)$ ) for a positive step and $A_{+}=-\left(12 F_{M}\right)^{1 / 2}$ (see(2.17)) for a negative step, which are exactly the same values of $A_{\mp}$ predicted by (2.1) for a localized obstacle. In this case the hydraulic limit predicts that there is no communication between the two ends of the step. Note that small-amplitude dispersive waves, in the fKdV equation (1.3) have a phase speed $c=\Delta+k^{2} / 6$ and a group velocity of $c_{g}=\Delta+k^{2} / 2$, where $k$ is the wavenumber. Such waves are not within the scope of the hydraulic limit, but may be generated as transients. With $\Delta=0$ as here, if present they can only propagate downstream. However, we see from Figure 1 that if such waves were generated, they must have very small amplitudes as they cannot be detected above the noise level in Figure 1. 


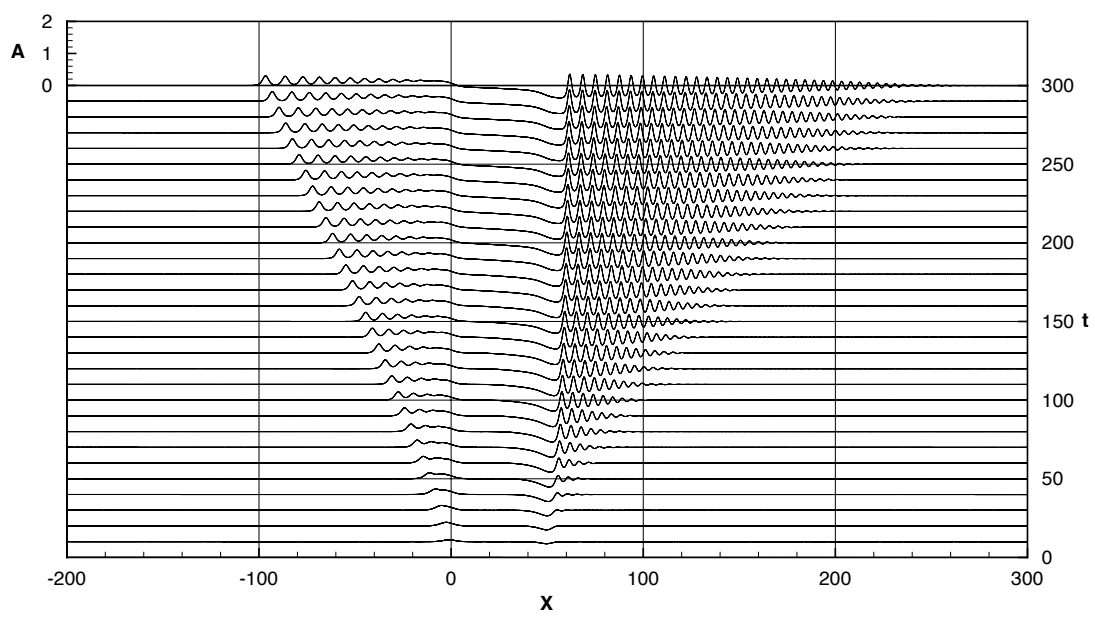

Figure 3. As for Figure 1, but with $\Delta=-0.2$

Next, for the supercritical case (Figure 2) we see there is again an upstream undular bore attached to the positive step at $x=0$, and a downstream undular bore propagating away from the negative step at $x=50$, leaving a depression zone behind. But now there is a positive rarefraction wave generated over the step, emanating from $x=0$. Again, this scenario is in complete accord with our theoretical predictions. Note that after taking account of the scalings (1.4) the parameter values for Figure 2 place the upstream solution in the regime (2.16) for an attached undular bore, here $-0.22<\Delta<0.39$, and the downstream solution in the regime (2.21) for a fully detached undular bore, here $\Delta>-0.19$. From Table 1 we see that there is again good agreement with the theoretical predictions, with the largest discrepancy again being for $A_{-}$. In this supercritical case, while the upstream and downstream solutions have the same qualitative structure as that for flow over a localized obstacle, the predicted values of $A_{\mp}$ are different. The scenario described above and depicted in Figure 2 persists until the rarefraction wave reaches the end of the step at $x=50$; since the leading edge of this rarefraction wave propagates with a speed $\Delta$, this will occur when $t \approx L / \Delta=250$. After this time, there will be an adjustment to the whole solution which will eventually settle down to the same solution as that described by Grimshaw \& Smyth (1986) for flow over a localized obstacle. As for the critical case, we cannot detect the presence of any transient small-amplitude dispersive waves, which if present must propagate downstream.

For the subcritical case (Figure 3) there is now a fully detached upstream bore, while the downstream bore has intensified and propagates more slowly, but is still fully detached. There is a negative rarefraction wave propagating upstream, emanating from $x=50$. Again, this scenario is in accord with our theoretical predictions. But we note that the parameter values of Figure 3 would place the upstream solution in the regime (2.16), here $-0.22<\Delta<0.39$, for an attached undular bore, and the downstream solution in regime $(2.22)$ (here $-0.38<\Delta<-0.19$ ) also for an attached undular bore. 


\begin{tabular}{|c|l|l|l|l|l|l|l|l|}
\hline & \multicolumn{4}{|c|}{ fKdV } & \multicolumn{4}{c|}{ Theory } \\
\cline { 2 - 9 }$\Delta$ & $\mathbf{A}_{\boldsymbol{w}}$ & $\mathbf{A}$ & $\mathbf{A}_{\mathbf{w}}$ & $\mathbf{A}_{+}$ & $\mathbf{A}_{\mathbf{w}}$ & $\mathbf{A}$ & $\mathbf{A}_{\mathbf{w}}$ & $\mathbf{A}_{+}$ \\
\hline $\mathbf{0 . 2}$ & $\mathbf{0 . 8 3}$ & $\mathbf{0 . 4 4}$ & $\mathbf{0 . 3 1}$ & $\mathbf{- 0 . 1 6}$ & $\mathbf{0 . 8 0}$ & $\mathbf{0 . 4 0}$ & $\mathbf{0 . 3 2}$ & $\mathbf{- 0 . 1 6}$ \\
\hline $\mathbf{0 . 1}$ & $\mathbf{0 . 6 6}$ & $\mathbf{0 . 3 8}$ & $\mathbf{0 . 3 9}$ & $\mathbf{- 0 . 2 0}$ & $\mathbf{0 . 6 6}$ & $\mathbf{0 . 3 3}$ & $\mathbf{0 . 4 0}$ & $\mathbf{- 0 . 2 0}$ \\
\hline $\mathbf{0 . 0}$ & $\mathbf{0 . 5 0}$ & $\mathbf{0 . 3 0}$ & $\mathbf{0 . 5 1}$ & $\mathbf{- 0 . 2 6}$ & $\mathbf{0 . 5 2}$ & $\mathbf{0 . 2 6}$ & $\mathbf{0 . 5 2}$ & $\mathbf{- 0 . 2 6}$ \\
\hline $\mathbf{- 0 . 1}$ & $\mathbf{0 . 3 9}$ & $\mathbf{0 . 2 2}$ & $\mathbf{0 . 6 4}$ & $\mathbf{- 0 . 3 3}$ & $\mathbf{0 . 4 0}$ & $\mathbf{0 . 2 0}$ & $\mathbf{0 . 6 6}$ & $\mathbf{- 0 . 3 3}$ \\
\hline $\mathbf{- 0 . 2}$ & $\mathbf{0 . 3 0}$ & $\mathbf{0 . 1 6}$ & $\mathbf{0 . 8 4}$ & $\mathbf{- 0 . 4 0}$ & $\mathbf{0 . 3 2}$ & $\mathbf{0 . 1 6}$ & $\mathbf{0 . 8 0}$ & $\mathbf{- 0 . 4 0}$ \\
\hline $\mathbf{- 0 . 3}$ & $\mathbf{0 . 2 4}$ & $\mathbf{0 . 1 3}$ & $\mathbf{0 . 6 4}$ & $\mathbf{- 0 . 3 8}$ & $\mathbf{0 . 2 6}$ & $\mathbf{0 . 1 3}$ & $\mathbf{0 . 9 2}$ & $\mathbf{- 0 . 4 6}$ \\
\hline $\mathbf{- 0 . 4}$ & $\mathbf{0 . 1 9}$ & $\mathbf{0 . 1 0}$ & $\mathbf{0 . 0 0}$ & $\mathbf{0 . 0 0}$ & $\mathbf{0 . 2 0}$ & $\mathbf{0 . 1 0}$ & $\mathbf{0 . 0 0}$ & $\mathbf{0 . 0 0}$ \\
\hline
\end{tabular}

\begin{tabular}{|c|c|c|c|c|c|c|c|c|}
\hline \multirow{3}{*}{$\Delta$} & \multicolumn{4}{|c|}{ fKdV } & \multicolumn{4}{c|}{ Euler } \\
\cline { 2 - 9 } & $\mathbf{A}_{\boldsymbol{w}-}$ & $\mathbf{A}$. & $\mathbf{A}_{\boldsymbol{w}+}$ & $\mathbf{A}_{+}$ & $\mathbf{A}_{\mathbf{w}-}$ & $\mathbf{A}$. & $\mathbf{A}_{\boldsymbol{w}+}$ & $\mathbf{A}_{+}$ \\
\hline $\mathbf{0 . 2}$ & $\mathbf{0 . 8 3}$ & $\mathbf{0 . 4 4}$ & $\mathbf{0 . 3 1}$ & $\mathbf{- 0 . 1 6}$ & $\mathbf{0 . 7 5}$ & $\mathbf{0 . 4 0}$ & $\mathbf{0 . 2 8}$ & $\mathbf{- 0 . 1 8}$ \\
\hline $\mathbf{0 . 1}$ & $\mathbf{0 . 6 6}$ & $\mathbf{0 . 3 8}$ & $\mathbf{0 . 3 9}$ & $\mathbf{- 0 . 2 0}$ & $\mathbf{0 . 5 7}$ & $\mathbf{0 . 3 6}$ & $\mathbf{0 . 3 2}$ & $\mathbf{- 0 . 2 1}$ \\
\hline $\mathbf{0 . 0}$ & $\mathbf{0 . 5 0}$ & $\mathbf{0 . 3 0}$ & $\mathbf{0 . 5 1}$ & $\mathbf{- 0 . 2 6}$ & $\mathbf{0 . 4 4}$ & $\mathbf{0 . 3 3}$ & $\mathbf{0 . 3 7}$ & $\mathbf{- 0 . 2 5}$ \\
\hline $\mathbf{- 0 . 1}$ & $\mathbf{0 . 3 9}$ & $\mathbf{0 . 2 2}$ & $\mathbf{0 . 6 4}$ & $\mathbf{- 0 . 3 3}$ & $\mathbf{0 . 3 2}$ & $\mathbf{0 . 2 0}$ & $\mathbf{0 . 4 3}$ & $\mathbf{- 0 . 3 0}$ \\
\hline $\mathbf{- 0 . 2}$ & $\mathbf{0 . 3 0}$ & $\mathbf{0 . 1 6}$ & $\mathbf{0 . 8 4}$ & $\mathbf{- 0 . 4 0}$ & $\mathbf{0 . 2 3}$ & $\mathbf{0 . 1 3}$ & $\mathbf{0 . 5 3}$ & $\mathbf{- 0 . 3 6}$ \\
\hline $\mathbf{- 0 . 3}$ & $\mathbf{0 . 2 4}$ & $\mathbf{0 . 1 3}$ & $\mathbf{0 . 6 4}$ & $\mathbf{- 0 . 3 8}$ & $\mathbf{0 . 1 6}$ & $\mathbf{0 . 0 8}$ & $\mathbf{0 . 5 7}$ & $\mathbf{- 0 . 3 8}$ \\
\hline $\mathbf{- 0 . 4}$ & $\mathbf{0 . 1 9}$ & $\mathbf{0 . 1 0}$ & $\mathbf{0 . 0 0}$ & $\mathbf{0 . 0 0}$ & $\mathbf{0 . 1 0}$ & $\mathbf{0 . 0 1}$ & $\mathbf{0 . 0 0}$ & $\mathbf{0 . 0 0}$ \\
\hline
\end{tabular}

TABLE 1. A quantitative comparison of the numerical simulations of the fKdV equation (1.3) and the Euler equations with the theory. $A_{-}\left(A_{+}\right)$is the elevation just upstream (downstream) of the positive (negative) step at $x=0(50)$ respectively, and $A_{w-}\left(A_{w+}\right)$ is the amplitude of the leading wave in the corresponding undular bore.

However, our numerical results for $\Delta=-0.2$ apparently place the upstream solution in the regime $(2.15)$ here $\Delta<-0.22$, for a detached undular bore, and the downstream solution in the regime (2.21), here $\Delta>-0.19$, again for a detached undular bore. But because the value of $\Delta$ is very close to the boundaries of these regime changes, we attribute this small discrepancy to errors in estimating $A_{\mp}$, and hence the regime boundaries, from the hydraulic limit. Nevertheless there is good quantitative agreement with our theoretical predictions, see Table 1. As for the supercritical case, although the upstream and downstream solutions have the same qualitative structure as that for flow over a localized obstacle, the quantitative values of $A_{\mp}$ are different. But again, the scenario described above and depicted in Figure 3 will only persist until the rarefraction wave reaches the end of the step at $x=0$; since the leading edge of this rarefraction wave propagates with a speed $-\Delta$, this will occur when $t \approx L / \Delta=250$. After this time, there will be an adjustment to the whole solution which will eventually settle down to the same solution as that described by Grimshaw \& Smyth (1986) for flow over a localized obstacle. 


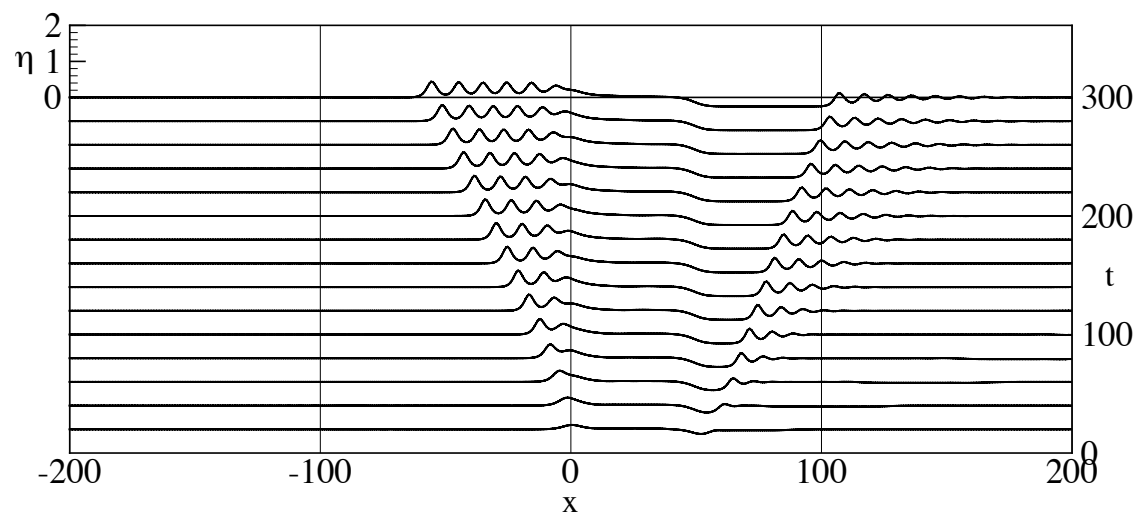

FiguRE 4. Numerical simulation of the Euler equations for $F_{M}=0.1, \gamma=0.25, L=50, \Delta=0.0$.

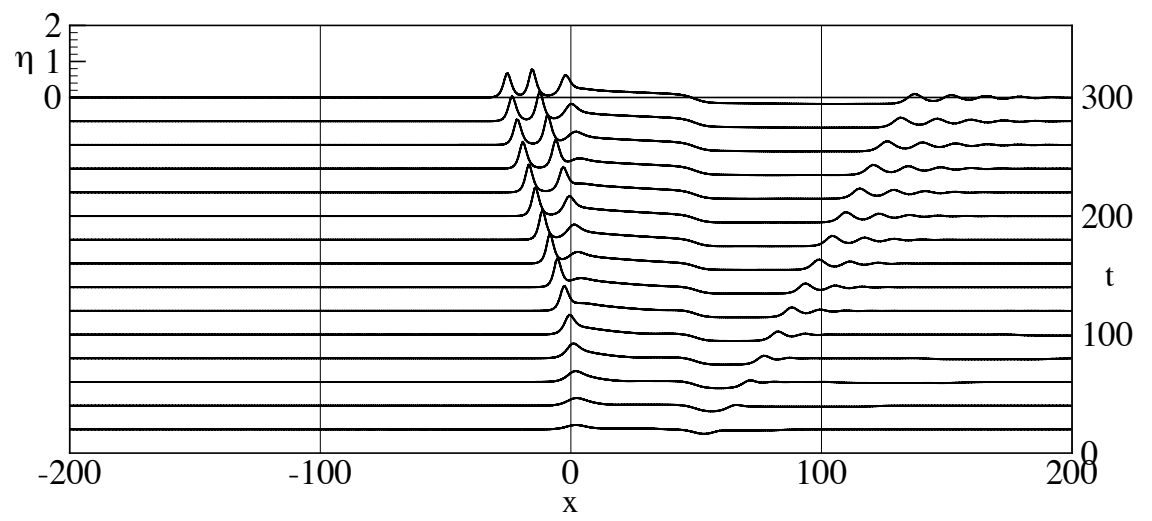

Figure 5. As for Figure 4, but with $\Delta=0.2$.

Again, as for the previous two cases, we cannot detect the presence of any transient small-amplitude dispersive waves, although in this subcritical case they can propagate upstream for wavenumbers $k<\sqrt{2|\Delta|}$ (note that the most likely wavenumber to be generated is $k \sim \gamma$ which for our parameter setting implies upstream propagation).

The corresponding results for the Euler equations are shown in Figures 4 to 6 . We see that there is always a good qualitative agreement, but as shown in Table 1, the quantitative results are different. The amplitudes of the leading upstream and downstream waves, and the amplitudes of the upstream elevation and the downstream depression are consistently smaller than the corresponding entries for the fKdV equation. We attribute this to the effect of nonlinearity, as when the forcing amplitude $F_{M}$ is reduced from 0.1 to 0.05 , we find that the quantitative agreement is significantly improved. In this con- 


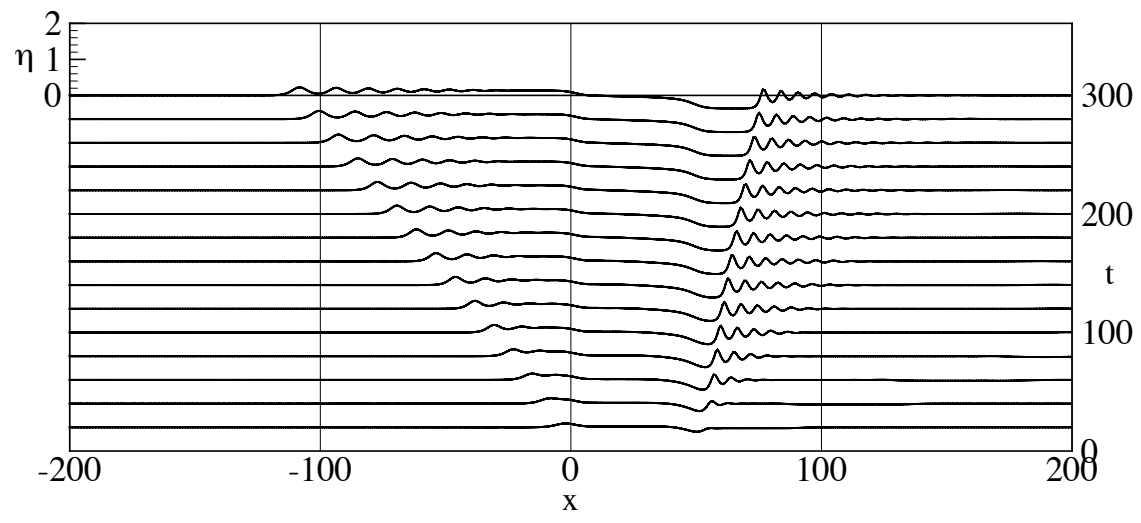

Figure 6. As for Figure 4, but with $\Delta=-0.2$.

text we note that a similar discrepancy was found by Zhang \& Chwang (2001) for the waves produced by flow over a localized obstacle. This discrepancy in the amplitudes also accounts for the different locations and speeds of the waves when comparing the fKdV simulations with the Euler equation simulations. Nevertheless, we can note that importantly the variation of all the predicted amplitudes and elevations as $\Delta$ is varied follow the same trend for both the fKdV and Euler equations. Further in all the Euler equation cases we simulated there was no evidence of any other wavetrains generated than those predicted by the fKdV equation. In particular, over the step itself, the only wave generated is a downstream (upstream) propagating rarefraction wave for $\Delta>0(<0)$, just as in the $\mathrm{fKdV}$ equation. We infer, at least for the small-amplitude steps we have considered here, that the fKdV equation with its upstream and downstream undular bores provides a very good guide for transcritical flow over a step.

\section{Conclusions}

In this paper, we have explored transcritical flow over a step primarily in the framework of the forced Korteweg-de Vries (fKdV) equation (1.5), using both asymptotic analysis and numerical simulations. Our results show that a positive step generates an upstreampropagating undular bore formed by a (stationary) elevation upstream of the step, and a negative step generates a downstream-propagating undular bore formed by a (stationary depression downstream of the step. The extent and strengths of the undular bores vary with the criticality parameter $\Delta$. Although our emphasis here has been on water waves, our results apply in many other physical contexts as the fKdV equation is a canonical model for transcritical flow past an obstacle. In the water wave context, direct simulations of the full Euler equations confirm the scenarios identifed here in the fKdV model, provided of course that the obstacle, and hence the waves generated, have sufficiently small amplitudes.

All the numerical results displayed here have been obtained for the obstacle (3.5) with $F_{M}>0$, that is, the flow encounters first a positive step, followed a long constant height section, which is then terminated by a negative step. However, the analogous results when $F_{M}<0$ in (3.5), that is, the flow encounters first a negative step, then a long 
constant height section terminated by a positive step, can be inferred from the results we have obtained. Thus, as discussed by Zhang \& Chwang (2001), we would expect to see a depression and a downstream-propagating undular bore form at the negative step, and an elevation and an upstream-propagating undular bore form at the positive step. But, unlike the case for $F_{M}>0$, with $F_{M}<0$ these undular bores will meet and interact over the step itself. Although an analysis of this interaction may be possible in the framework of the Whitham equations (see Appendix A), we shall not attempt this quite daunting task. Instead we note that numerical simulations of the fKdV equation for a negative obstacle by Grimshaw \& Smyth (1986) and others, and the numerical simulations of the Euler equations by Zhang \& Chwang (2001) indicate that the outcome of this interaction is again upstream-propagating and downstream-propagating undular bores, but the solution over the obstacle or step remains unsteady.

Finally, we have already noted that the long-time solution for flow over a step of finite length (that is, (3.5) for instance with $F_{M}>0, \gamma L>>1$ ) will be that predicted by Grimshaw \& Smyth (1986) in the framework of the fKdV equation for flow over a localised obstacle. Indeed, at exact criticality $\Delta$, the wavetrains generated by the elongated step are in fact exactly the same as those predicted for flow over a localised obstacle. Otherwise, for $\Delta \neq 0$, the upstream and downstream undular bores initially generated by the positive and negative steps have (slightly) different amplitudes to those generated by a localized obstacle, but for sufficiently long times $(t>L /|\Delta|$ there is communication between the two steps by a rarefraction wave, followed by an adjustment to precisely the same solution predicted by Grimshaw \& Smyth (1986).

Partial financial support for this work was provided by the Research Grants Council of Hong Kong through contracts HKU 7123/05E.

\section{Appendix A. Undular bore}

The term "undular bore" is widely used in the literature in a variety of contexts and several different meanings. Here, we need to make it clear that we are concerned with non-dissipative flows, in which case an undular bore is intrinsically unsteady. In general, an undular bore is an oscillatory transition between two different basic states. A simple representation of an undular bore can be obtained from the solution of the KdV equation

$$
A_{t}+6 A A_{x}+A_{x x x}=0 .
$$

with the initial condition that

$$
A=A_{0} H(-x),
$$

where we assume at first that $A_{0}>0$. Here $H(x)$ is the Heaviside function (i.e. $H(x)=1$ if $x>0$ and $H(x)=0$ if $x<0)$. The solution can in principle be obtained through the inverse scattering transform. However, it is more instructive to use the asymptotic method developed by Gurevich \&Pitaevskii (1974) and Whitham (1974). In this approach, the solution of (A 1) with this initial condition is represented as the modulated periodic wave train

$$
\begin{aligned}
A & =a\left\{b(m)+\mathrm{cn}^{2}(\kappa(x-V t) ; m)\right\}+d, \\
\text { where } \quad b & =\frac{1-m}{m}-\frac{E(m)}{m K(m)}, \quad a=2 m \kappa^{2}, \\
\text { and } \quad V & =6 d+2 a\left\{\frac{2-m}{m}-\frac{3 E(m)}{m K(m)}\right\} .
\end{aligned}
$$


Here $\operatorname{cn}(x ; m)$ is the Jacobian elliptic function of modulus $m$ and $K(m), E(m)$ are the elliptic integrals of the first and second kind respectively. $(0<m<1), a$ is the wave amplitude, $d$ is the mean level, and $V$ is the wave speed. The spatial period is $2 K(m) / \kappa$. This family of solutions contains three free parameters, which are chosen from the set $(a, \kappa, V, d, m)$. As $m \rightarrow 1, \operatorname{cn}(x \mid m) \rightarrow \operatorname{sech}(x)$ and then the cnoidal wave (A 3) becomes a solitary wave, riding on a background level $d$. On the other hand, as $m \rightarrow 0, \operatorname{cn}(x \mid m) \rightarrow$ $\cos 2 x$ and so the cnoidal wave (A 3 ) collapses to a linear sinusoidal wave (note that in this limit $a \rightarrow 0$ ).

The asymptotic method of Gurevich and Pitaevskii (1974) and Whitham (1974) is to let the expression (A 3) describe a modulated periodic wavetrain in which the amplitude $a$, the mean level $d$, the speed $V$ and the wavenumber $\kappa$ are all slowly varying functions of $x$ and $t$. The outcome is a set of three nonlinear hyperbolic equations for three of the available free parameters, chosen from the set $(a, \kappa, V, d, m)$, or rather better, from an appropriate combinations of them. These equations are often called the Whitham equations. The relevant asymptotic solution corresponding to the initial condition (A 2) is then constructed in terms of the similarity variable $x / t$, and is given by

$$
\begin{gathered}
\frac{x}{t}=2 A_{0}\left\{1+m-\frac{2 m(1-m) K(m)}{E(m)-(1-m) K(m)}\right\}, \\
\text { for } \quad-6 A_{0}<\frac{x}{t}<4 A_{0}, \\
a=2 A_{0} m, \quad d=A_{0}\left\{m-1+\frac{2 E(m)}{K(m)}\right\} .
\end{gathered}
$$

Ahead of the wavetrain where $x / t>4 A_{0}, A=0$ and at this end, $m \rightarrow 1, a \rightarrow 2 A_{0}$ and $d \rightarrow 0$; the leading wave is a solitary wave of amplitude $2 A_{0}$ relative to a mean level of 0 . Behind the wavetrain where $x / t<-6 A_{0}, A=A_{0}$ and at this end $m \rightarrow 0, a \rightarrow 0$, and $d \rightarrow A_{0}$; the wavetrain is now sinusoidal with a wavenumber $\kappa$ given by $6 \kappa^{2} \approx A_{0}$. Further, it can be shown that on any individual crest in the wavetrain, $m \rightarrow 1$ as $t \rightarrow \infty$. In this sense, the undular bore evolves into a train of solitary waves.

If $A_{0}<0$ in the initial condition (A 3), then an "undular bore" solution analogous to that described by (A 3, A 5) does not exist. Instead, the asymptotic solution is a rarefraction wave,

$$
\begin{aligned}
& A=0 \quad \text { for } \quad x>0, \\
& A=\frac{x}{6 t} \quad \text { for } \quad A_{0}<\frac{x}{6 t}<0, \\
& A=A_{0}, \quad \text { for } \quad \frac{x}{6 t}<A_{0}(<0) .
\end{aligned}
$$

Small oscillatory wavetrains are needed to smooth out the discontinuities in $A_{x}$ at $x=0$ and $x=-6 A_{0}$, (see Gurevich \&Pitaevskii 1974).

\section{REFERENCES}

AKYlas, T.R. (1984) On the excitation of long nonlinear water waves by moving pressure distribution. J.Fluid Mech., 141, 455-466.

Cole, S. L. (1985). Transient waves produced by flow past a bump. Wave Motion , 7, 579-587.

Ertekin, R. C., Webster, W. C. \& Wehausen, J. V. (1984) Ship generated solitons. Proc. 15th Symp. Naval Hydrodyn., Hamburg, 347-364.

Ertekin, R. C., Webster, W. C. \& Wehausen, J. V. (1986) Waves caused by a moving disturbance in a shallow channel of finite width. J. Fluid Mech., 169: 275-292 
Grimshaw, R. H. J. \& Smyth, N. F. 1986 Resonant flow of a stratified fluid over topography. J. Fluid Mech. 169, 429-464.

Gurevich, A. V. \& Pitaevskit, L. P. 1974 Nonstationary structure of a collisionless shock wave. Sov. Phys. JETP 38, 291-297.

Huang, D. B., Sibul, O. J., Webster, W.C., Wehausen, J. V., Wu, D. M. \& Wu, T. Y. (1982) Ships moving in the transcritical range. Proc. Conf. on Behaviour of Ships in Restricted Waters, Varna, Bulgaria, 26-1-26-10.

King, A. C. And Bloor, M. I. G. (1987). Free-surface flow over a step. J. Fluid Mech., 182, 193-208.

Lee S. J., Yates G. T., \& Wu T. Y. (1989). Experiments and analyses of upstream-advancing solitary waves generated by moving disturbances. J. Fluid Mech., 199, 569-593.

Lighthill, James 1978 Waves in Fluids, CUP.

MEI, C. C. (1986) Radiation of solitons by slender bodies advancing in a shallow channel. $J$. Fluid Mech. 162: 53-67.

Melville, W.K. \& Helfrich K. R. (1987) Transcritical two-layer flow over topography. J.Fluid Mech., 178, 31-52.

Smyth, N. F. (1987) Modulation theory for resonant flow over topography, Proc. Roy. Soc. 409A, 79-97.

The influence of Shallow WATER on the RESistance of A CRUiser MOdel, A ThIRTYINCH MODEL OF THE S.S. ClairTON IN SHALlOW WATER. US Experimental Model Basin, Washington, D.C., Rep. 408, 414.

Whitham, G. B.1974 Linear and Nonlinear Waves, Wiley.

Wu T.Y. (1987). Generation of upstream advancing solitons by moving disturbances . J. Fluid Mech., 184, 75-99.

Wu, D. M. \& Wu, T. Y. (1982) Three-dimensional nonlinear long waves due to moving surface pressure. Proc. 14th Symp. Naval Hydrodyn., Washington, 103-129.

Zhang, D.-H. \& ChwANG, A. T. 1999 On solitary waves forced by underwater moving objects. J. Fluid Mech. 389, 119-135.

Zhang, D.-H. \& Chwang, A. T. 2001 Generation of solitary waves by forward- and backwardstep bottom forcing. J. Fluid Mech. 432, 341-350. 